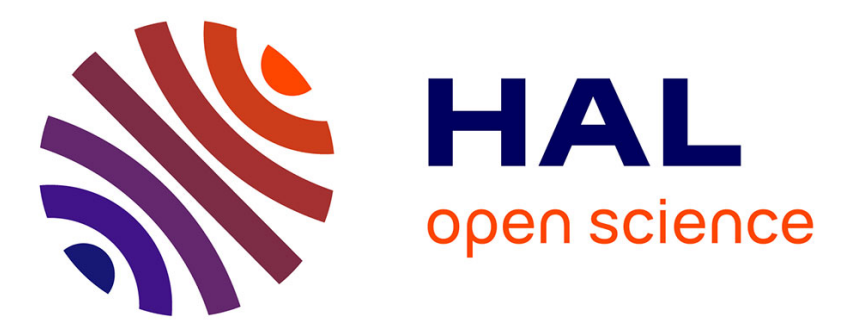

\title{
Cristallochimie du sélénium(+IV). I. VSe2O6, une structure à trois chaines parallèles (VO5)6n- indépendantes pontées par des groupements $(\mathrm{Se} 2 \mathrm{O}) 6+$ Georges Meunier, Michel Bertaud, Jean Galy
}

\section{- To cite this version:}

Georges Meunier, Michel Bertaud, Jean Galy. Cristallochimie du sélénium(+IV). I. VSe2O6, une structure à trois chaines parallèles (VO5)6n- indépendantes pontées par des groupements (Se2O)6+. Acta crystallographica Section B: Structural crystallography and crystal chemistry, 1974, 30, pp.28342839. 10.1107/S0567740874008260 . hal-00147390

\section{HAL Id: hal-00147390 \\ https://hal.science/hal-00147390}

Submitted on 16 May 2007

HAL is a multi-disciplinary open access archive for the deposit and dissemination of scientific research documents, whether they are published or not. The documents may come from teaching and research institutions in France or abroad, or from public or private research centers.
L'archive ouverte pluridisciplinaire HAL, est destinée au dépôt et à la diffusion de documents scientifiques de niveau recherche, publiés ou non, émanant des établissements d'enseignement et de recherche français ou étrangers, des laboratoires publics ou privés. 
Acta Crystallographica Section B

Structural

Science

ISSN 0108-7681

Editor: Carolyn P. Brock

Cristallochimie du sélénium(+IV). I. $\mathrm{VSe}_{2} \mathrm{O}_{6}$, une structure à trois chaines parallèles $\left(\mathrm{VO}_{5}\right)^{6 n-}$ indépendantes pontées par des groupements $\left(\mathrm{Se}_{2} \mathrm{O}\right)^{6+}$

G. Meunier, M. Bertaud et J. Galy

Copyright $($ International Union of Crystallography

Author(s) of this paper may load this reprint on their own web site provided that this cover page is retained. Republication of this article or its storage in electronic databases or the like is not permitted without prior permission in writing from the IUCr. 


\section{References}

BART, J. C. J. \& Schmidt, G. M. J. (1971). Personal communication.

Berghuis, J., HaAnappel, IJ. M., Potters, M., Loopstra, B. O., MacGillavry, C. H. \& VeenendaAl, A. L. (1955). Acta Cryst. 8, 478-483.

BoIs, C. (1970). Acta Cryst. B26, 2086-2092.

Buckles, R. E., Hausman, E. A. \& Wheeler, N. G. (1950). J. Amer. Chem. Soc. 72, 2494-2496.

Carter, O. L., McPhail, A. T. \& Sim, G. A. (1966). $J$. Chem. Soc. $(A)$, pp. 822-827.

Casalone, G., Gavezzotti, A., Mariani, C., Mugnoli, A. \& SimonetTa, M. (1970). Acta Cryst. B 26, 1-8.

Filippakis, S. E., Leiserowitz, L. \& Schmidt, G. M. J. (1967). J. Chem. Soc. (B), pp. 290-296.

Germain, G. \& Woolfson, M. M. (1968). Acta Cryst. B24, 91-96.

Hadjoudis, E., Kariv, E. \& Schmidt, G. M. J. (1972). $J$ Chem. Soc. Perkin II, pp. 1056-1060.

Hope, H. (1969). Acta Cryst. B 25, 78-87.

JOHNSON, C. K. (1965). ORTEP. Report ORNL-3794, Oak Ridge National Laboratory, Oak Ridge, Tennessee.

Jungk, A. E. \& Schmidt, G. M. J. (1970). J. Chem. Soc. (B), pp. 1427-1434.

Kato, K. (1971). Acta Cryst. B 27, 2028-2034.

Kaufman, H. W. \& Leiserowitz, L. (1970). Acta Cryst. B26, 442-443.

Labes, M. M., Blakeslee, H. W. \& Bloor, J. E. (1965). J. Amer. Chem. Soc. 87, 4251-4255.

Leiserowitz, L. \& Rabinovich, D. (1969). J. Chem. Soc. $(A)$, pp. 2367-2371.
MCWEENY, R. (1951). Acta Cryst. 4, 513-519.

Miller, R. S., Curtin, D. Y. \& Paul, I. C. (1971). J. Amer. Chem. Soc. 93, 2784-2786.

Miller, R. S., Curtin, D. Y. \& Paul, I. C. (1972). J. Amer. Chem. Soc. 94, 5117-5119.

Penzien, K. \& Schmidt, G. M. J. (1969a). Angew. Chem. Int. Ed. 8, 608-609.

Penzien, K. \& Schmidt, G. M. J. (1969b). Personal communication.

Rabinovich, D. (1969). J. Chem. Soc. (A), pp. 23612366.

Rabinovich, D. (1970). J. Chem. Soc. (B), pp. 11-16.

RABINOVICH, D. (1974). In preparation.

Rabinovich, D. \& Schmidt, G. M. J. (1970). J. Chem. Soc. (B), pp. 6-10.

Rabinovich, D., Schmidt, G. M. J. \& Shakked, Z. (1970). J. Chem. Soc. (B), pp. 17-24.

Rabinovich, D., SchmidT, G. M. J. \& SHAKKED, Z. (1973). J. Chem. Soc. Perkin II, pp. 33-37.

Schomaker, V. \& Trueblood, K. N. (1968). Acta Cryst. B24, 63-76.

SHMUELI, U. (1972). Program TMA. Rigid-Body Motion Analysis, Department of Chemistry, Tel-Aviv Univ., TelAviv, Israel.

Smith, P., Florencio, F. \& García-Blanco, S. (1971). Acta Cryst. B 27, 2255-2260.

Takwale, M. G. \& Pant, L. M. (1971). Acta Cryst. B27, $1152-1158$

Warshel, A., Huller, E., Rabinovich, D. \& Shakked, Z. (1974). J. Mol. Struct. In the press.

Warshel, A. \& Karplus, M. (1972). J. Amer. Chem. Soc. 94, 5612-5625.

Acta Cryst. (1974). B30, 2834

\title{
Cristallochimie du Sélénium(+ IV). I. $\mathrm{VSe}_{2} \mathrm{O}_{6}$, une Structure à Trois Chaînes Parallèles $\left(\mathrm{VO}_{5}\right)_{n}^{6 n-}$ Indépendantes Pontées par des Groupements $\left(\mathrm{Se}_{2} \mathrm{O}\right)^{6+}$
}

\author{
Par Georges Meunier, Michel Bertaud et Jean Galy \\ Service de Chimie Minérale Structurale de l'Université de Bordeaux I, associé au CNRS, 351 Cours \\ de la Libération, 33405-Talence, France
}

(Reçu le 17 mai 1974, accepté le 11 juillet 1974)

\begin{abstract}
$\mathrm{VSe}_{2} \mathrm{O}_{6}$, a new compound prepared by solid-state reaction, crystallizes as pale-green blocks. The unit cell is tetragonal with the dimensions: $a=11.22$ and $c=7.86 \AA$; the space group is $P 4 c c$. The structure, determined by Patterson and Fourier methods, has been refined by least-squares procedures to an $R$ of 0.041 . The structure contains three independent $\left(\mathrm{VO}_{5}\right)_{n}^{6 n-}$ strings of octahedra sharing corners parallel to the [001] direction; the three strings are connected to each other by $\left(\mathrm{Se}_{2} \mathrm{O}\right)^{6+}$ groups. In the $\mathrm{VO}_{6}$ octahedra the vanadium atoms are off-centred forming $\mathrm{VO}^{2+}$ vanadyl groups. The coordination of selenium( + IV) is 3 , the lone pair $E$ forming with the oxygen atoms a tetrahedron. $\left(\mathrm{Se}_{2} \mathrm{O}_{5}\right)^{2-}$ groups appear in this structure and their stereochemistry is compared with $\left(\mathrm{Sn}_{2} \mathrm{~F}_{5}\right)^{-}$groups of the double fluoride $\mathrm{NaSn}_{2} \mathrm{~F}_{5}$.
\end{abstract}

Voici quelques années un travail d'ensemble était entrepris au laboratoire sur la cristallochimie des composés oxygénés du tellure (+IV) (Meunier \& Galy, 1971; Galy \& Meunier, 1971; Meunier, Darriet \& Galy, 1972, 1973; Galy, 1972). L'activité stéréochi- mique de la paire non liée $5 s_{5}$ du tellure $(+I V)$ a été analysée et son influence, au sein des nombreuses phases étudiées, précisée (Andersson, Åström, Galy \& Meunier, 1973).

Il paraissait particulièrement intéressant d'étendre 
ces investigations au sélénium(+IV) de configuration électronique [Ar] $3 d^{10} 4 s^{2}$. Par ailleurs, la chimie et la cristallochimie des composés oxygénés du sélénium(+IV) est actuellement fort peu connue.

Ce mémoire rapporte l'étude chimique et structurale d'un des systèmes pseudo binaires envisagés: $\mathrm{SeO}_{2}-$ $\mathrm{VO}_{2}$ (dioxyde de vanadium).

\section{Etude chimique du système $\mathrm{SeO}_{2}-\mathrm{VO}_{2}$}

\section{Synthèses}

Par analyse radiocristallographique une seule phase de formule $\mathrm{VSe}_{2} \mathrm{O}_{6}$ a été mise en évidence; elle correspond au rapport molaire $\mathrm{SeO}_{2} / \mathrm{VO}_{2}=2$.

Cette phase s'obtient pure par interaction directe des oxydes $\mathrm{SeO}_{2}$ et $\mathrm{VO}_{2}$, selon la réaction:

$$
2 \mathrm{SeO}_{2}+\mathrm{VO}_{2} \rightarrow \mathrm{VSe}_{2} \mathrm{O}_{6}
$$

Les oxydes $\mathrm{SeO}_{2}$ et $\mathrm{VO}_{2}$ finement pulvérisés sont longuement mélangés puis introduits en tube de Vycor; ce dernier est ensuite scellé. La réaction est totale après un traitement thermique de $24 \mathrm{~h}$ à $400^{\circ} \mathrm{C}$.

Le dioxyde de sélénium de départ est un produit commercial; il se présente sous forme d'aiguilles brillantes; mais étant légèrement hygroscopiques, les cristaux se recouvrent d'une mince pellicule d'acide sélénieux $\mathrm{H}_{2} \mathrm{SeO}_{3} . \mathrm{SeO}_{2}$ parfaitement anhydre s'obtient en desséchant le produit commercial à une température de l'ordre de $200^{\circ} \mathrm{C}$.

L'oxyde $\mathrm{VO}_{2}$ est obtenu à $850^{\circ} \mathrm{C}$ sous courant d'azote $\mathrm{R}$ désoxygéné, par interaction de quantités stoéchiométriques d'anhydride vanadique $\mathrm{V}_{2} \mathrm{O}_{5}$ et de sesquioxyde $\mathrm{V}_{2} \mathrm{O}_{3} . \mathrm{V}_{2} \mathrm{O}_{3}$ est préparé par réduction de $\mathrm{V}_{2} \mathrm{O}_{5}$ sous courant d'hydrogène à $800^{\circ} \mathrm{C}$.

$\mathrm{VSe}_{2} \mathrm{O}_{6}$ se présente sous forme d'une poudre vert clair bien cristallisée. Le spectre $\mathrm{X}$ de poudre de cette phase est consigné au Tableau 1.

Remarque: - Lorsque le rapport molaire $\mathrm{SeO}_{2} / \mathrm{VO}_{2}$ devient inférieur à 1 un phénomène d'oxydo-réduction apparaît avec formation de sélénium élémentaire.

Tableau 1. Indexation du spectre $X$ de poudre de $\mathrm{VSe}_{2} \mathrm{O}_{6}$

\begin{tabular}{|c|c|c|c|c|c|c|c|}
\hline$h k l$ & $d_{\text {obs. }}(\AA$ & calc. $(\AA \AA$ & $I / I_{o}$ & $h k l$ & $d_{\text {obs. }}(\AA)$ & $d_{\text {calc. }}(\AA)$ & \\
\hline $\begin{array}{lll}1 & 1 & 0\end{array}$ & $\begin{array}{l}7,93 \\
4,23\end{array}$ & $\begin{array}{l}7,93 \\
4,23\end{array}$ & $\begin{array}{r}<2 \\
7\end{array}$ & $\begin{array}{lll}1 & 2 & 3 \\
0 & 4 & 2\end{array}$ & & $\begin{array}{l}2,323 \\
2,284\end{array}$ & \\
\hline & 3,966 & 3,967 & 24 & & 2,244 & 2,244 & \\
\hline 02 & 3,931 & 3,9 & 60 & & & & \\
\hline & $\begin{array}{l}3,7 \\
3,5\end{array}$ & $\begin{array}{l}3,7 \\
3,5\end{array}$ & $\begin{array}{r}<2 \\
4\end{array}$ & & 59 & & \\
\hline & 3,2 & 3,2 & 100 & & 2,117 & 2, & \\
\hline & 3,0 & 3,0 & 20 & & 5 & & \\
\hline & 2,8 & 2,8 & 4 & & & & \\
\hline & 2,8 & 2,8 & $<2$ & & & & \\
\hline & 2,7 & 2,7 & 10 & & & & \\
\hline & 2,6 & & 10 & & & & \\
\hline & & & 32. & & & & \\
\hline & & & $<2$ & & & 1,6 & \\
\hline
\end{tabular}

\section{Dosages}

L'oxyde de sélénium $\mathrm{SeO}_{2}$, de par sa température de sublimation très basse $\left(350^{\circ} \mathrm{C}\right)$, a tendance à se transporter à l'extrémité froide du tube réactionnel; aussi, la formule $\mathrm{VSe}_{2} \mathrm{O}_{6}$ a-t-elle été confirmée par dosage chimique du sélénium et du vanadium (Charlot, 1961).

Le sélénium est dosé par gravimétrie. Une masse connue de poudre est mise en solution à l'aide de l'acide chlorhydrique; celle-ci saturée par l'anhydride sulfureux est ensuite portée à ébullition. Le sélénium élémentaire précipite selon la réaction:

$$
2 \mathrm{SO}_{2}+\mathrm{H}_{2} \mathrm{O}+\mathrm{H}_{2} \mathrm{SeO}_{3} \rightarrow 2 \mathrm{H}_{2} \mathrm{SO}_{4}+\mathrm{Se} .
$$

Le précipité est lavé à l'eau, puis à l'alcool absolu, séché à l'étuve et pesé.

Sur une deuxième prise, le vanadium est oxydé à l'état $(+V)$ par l'acide perchlorique. Le dosage du $\mathrm{V}^{5}+$ ainsi formé est effectué par réduction à l'état (+IV), en milieu sulfurique par le fer ferreux. L'excès de $\mathrm{Fe}^{2+}$ est dosé en retour par le bichromate de potassium.

Les résultats de ces dosages sont donnés au Tableau 2.

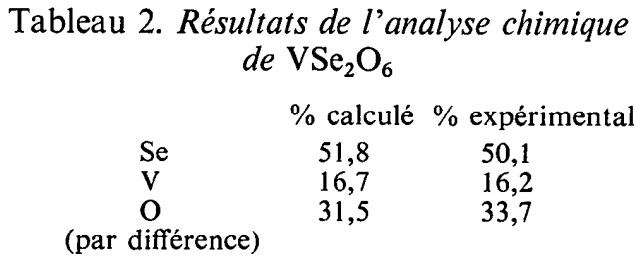

\section{Monocristaux}

De beaux monocristaux ont été obtenus après plusieurs jours de réaction à $350^{\circ} \mathrm{C}$ de la phase $\mathrm{VSe}_{2} \mathrm{O}_{6}$ en présence d'un excès de $\mathrm{SeO}_{2}$.

\section{Obtention d'une phase vitreuse}

La trempe brutale du liquide, obtenu après fusion à $550^{\circ} \mathrm{C}$ d'une poudre de composition $\mathrm{VSe}_{2} \mathrm{O}_{6}$ conduit à une masse lisse et homogène de couleur foncée. Après broyage, la poudre correspondante présente un spectre $X$ totalement exempt de raies, caractéristique d'une phase vitreuse.

\section{Etude radiocristallographique}

Les diagrammes de Bragg et de Weissenberg d'un monocristal de $\mathrm{VSe}_{2} \mathrm{O}_{6}$ ont révélé une symétrie quadratique et permis de préciser les paramètres de la maille (Tableau 3).

Tableau 3. Données cristallographiques de $\mathrm{VSe}_{2} \mathrm{O}_{6}$

$\begin{array}{ll}\text { Symétrie } & \text { Quadratique } \\ \text { Paramètres } & a=11,22 \pm 0,06 \AA \\ & c=7,86 \pm 0,04 \\ \text { Groupe spatial } & P 4 c c \\ d_{\text {exp. }} & 4,06 \pm 0,04 \mathrm{~g} \mathrm{~cm}^{-3} \\ d_{\text {catc. }} & 4,09 \\ Z & 8\end{array}$


Les règles d'extinction suivantes ont été relevées: $0 k l, l=2 n+1 ; h h l, l=2 n+1$; elles conduisent aux groupes spatiaux $P 4 c c$ ou $P 4 / m c c$.

A partir de ces données l'indexation du spectre $X$ de poudre a été effectuée et les paramètres de la maille cristalline affinés (Tableau 3).

La densité mesurée par pycnométrie dans l'orthophtalate de diéthyle est $d_{\text {exp. }}=4,06 \pm 0,04$; elle est en bon accord avec la densité calculée $\left(d_{x}=4,09\right)$ pour 8 motifs $\mathrm{VSe}_{2} \mathrm{O}_{6}$ par maille.

\section{Détermination de la structure}

Le monocristal choisi pour l'étude structurale se présente sous la forme d'un prisme régulier de dimensions $0,11 \times 0,10 \times 0,08 \mathrm{~mm}$.

Les intensités des réflexions $h k l$ ont été mesurées à l'aide d'un diffractomètre automatique Enraf Nonius CAD 3 (radiation Mo $K \alpha$, monochromateur à lame de graphite). L'angle maximum de mesure était $\theta=30^{\circ}$ (balayage en $\theta$ ). Seules ont été retenues les réflexions $h k l$ dont l'intensité satisfait au test $I_{h k l}>1,5 \sigma I_{h k l}$. Les 522 réflexions indépendantes sélectionnées ont été corrigées du facteur de Lorentz-polarisation.

Les facteurs de diffusion des atomes de sélénium, de vanadium et d'oxygène ont été relevés dans les tables de McMaster, Kerr del Grande, Mallet \& Hubbel (1969).

Les calculs ont été effectués sur IRIS 80 à l'aide de programmes mis au point au laboratoire par M. Saux.

Par déconvolution de la fonction de Patterson $P(u, v, w)$, deux points importants de la structure de $\mathrm{VSe}_{2} \mathrm{O}_{6}$ peuvent être précisés:

- le groupe spatial;

- la position des atomes lourds (Se et V).

Deux groupes spatiaux sont en effet possibles $P 4 c c$ (non centrosymétrique) et $P 4 / m c c$ (centrosymétrique). Les tests statistiques de Wilson (1949), de Howells, Philips \& Rogers (1950) et de Srinivasan (1960) plaident en faveur du groupe $P 4 c c$. Compte tenu par ailleurs des différences de côtes entre certains pics de la fonction $P(u, v, w)$ cette hypothèse se trouve confirmée.

Le test de piézoélectricité se révélant positif, le groupe spatial $P 4 c c$ est alors définitivement retenu.

Les positions des atomes lourds, déduites de la fonction de Patterson, sont les suivantes (16 séléniums et 8 vanadiums dans la maille):

\begin{tabular}{lrrrr} 
& & $x$ & $y$ & \multicolumn{1}{c}{$z$} \\
$\mathrm{Se}(1)$ & en $8(d)$ & 0,10 & 0,24 & 0 \\
$\mathrm{Se}(2)$ en $8(d)$ & 0,37 & 0,28 & 0,10 \\
$\mathrm{~V}(1)$ en $2(a)$ & 0 & 0 & 0,17 \\
$\mathrm{~V}(2)$ en $2(b)$ & $\frac{1}{2}$ & $\frac{1}{2}$ & 0,43 \\
$\mathrm{~V}(3)$ en $4(c)$ & 0 & $\frac{1}{2}$ & 0,10
\end{tabular}

La coordonnée $z$ de l'atome $\operatorname{Se}(1)$ a été fixée à la valeur $z=0$, afin de donner une origine suivant la direction de l'axe $O z$.
Après quelques cycles d'affinement, le facteur $R$ est de 0,157 .

Appliquant alors la méthode de l'atome lourd une synthèse de Fourier $\varrho(x y z)$ a été calculée; son étude détaillée permet de déduire les coordonnées approchées de huit atomes d'oxygène en positions indépendantes.

Après plusieurs cycles d'affinements, le facteur $R$ s'abaisse jusqu'à la très faible valeur $R=0,041$, confirmant ainsi le bien fondé des hypothèses successives. La fonction différence finale ne laisse apparaître aucun pic résiduel significatif.*

Tableau 4. Coordonnées réduites et paramètres d'agitation thermique isotrope avec leurs déviations standards (entre parenthèses)

\begin{tabular}{|c|c|c|c|c|c|}
\hline & Position & $x$ & $v$ & $z$ & $B\left(\AA^{2}\right)$ \\
\hline $\operatorname{Se}(1)$ & $8(d)$ & 0,1033 (1) & 0,2405 & 0 & $0,58(1)$ \\
\hline $\operatorname{Se}(2)$ & $8(d)$ & 0,3671 (1) & 0,2818 (1) & $0,1031(2)$ & 0,54 (1) \\
\hline$V(1)$ & $2(a)$ & 0 & 0 & $15(6)$ & $0,41(5)$ \\
\hline$V(2)$ & $2(b)$ & $\frac{1}{2}$ & $\frac{1}{2}$ & $4(5)$ & $0,28(5)$ \\
\hline$V(3)$ & $4(c)$ & 0 & $\frac{1}{2}$ & 72 (4) & $0,46(3)$ \\
\hline $\mathrm{O}(1)$ & $2(a)$ & 0 & 0 & $7(3)$ & $1,17(25)$ \\
\hline $\mathrm{O}(2)$ & $2(b)$ & $\frac{1}{2}$ & $\frac{1}{2}$ & $0,223(2)$ & $0,89(25)$ \\
\hline$O(3)$ & $4(c)$ & 0 & $\frac{1}{2}$ & 0,391 (1) & $1,00(17)$ \\
\hline$O(4)$ & $8(d)$ & $0,1233(7)$ & $0,1200(8)$ & $0,130(1)$ & 0,69 (11) \\
\hline $\mathrm{O}(5)$ & $8(d)$ & $0,2546(7)$ & $0,2550(7)$ & $0,440(1)$ & $0,68(10)$ \\
\hline$O(6)$ & $8(d)$ & $0,0919(7)$ & $0,3511(7)$ & 0,146 (1) & 0,81 (13) \\
\hline$O(7)$ & $8(d)$ & $0,3271(7)$ & $0,4759(7)$ & 0,468 (1) & $0,50(12)$ \\
\hline $\mathrm{O}(8)$ & $8(d)$ & $0,4059(7)$ & $0,1432(7)$ & $0,150(1)$ & $0,93(13)$ \\
\hline
\end{tabular}

Tableau 5. Distances $(\AA)$ et angles $\left(^{\circ}\right)$ interatomiques

$\begin{array}{lcll}\mathrm{Se}(1)-\mathrm{Se}(2) & 3,104(1) & \mathrm{V}(3)-\mathrm{O}(31) & 1,621(4) \\ \mathrm{Se}(1)-\mathrm{V}(1) & 3,232(2) & \mathrm{V}(3)-\mathrm{O}(81) & 1,967(9) \\ \mathrm{Se}(1)-\mathrm{V}(3) & 3,225(2) & \mathrm{V}(3)-\mathrm{O}(6) & 2,002(8) \\ \mathrm{Se}(2)-\mathrm{V}(2) & 3,867(2) & \mathrm{V}(3)-\mathrm{O}(3) & 2,311(4) \\ \mathrm{Se}(1)-\mathrm{O}(6) & 1,697(8) & \mathrm{O}(4)-\mathrm{O}(6) & 2,619(11) \\ \mathrm{Se}(1)-\mathrm{O}(4) & 1,710(8) & \mathrm{O}(4)-\mathrm{O}(51) & 2,586(11) \\ \mathrm{Se}(1)-\mathrm{O}(51) & 1,768(8) & \mathrm{O}(6)-\mathrm{O}(51) & 2,667(11) \\ \mathrm{Se}(1)-\mathrm{O}(41) & 3,056(8) & \mathrm{O}(8)-\mathrm{O}(51) & 2,678(12) \\ \mathrm{Se}(1)-\mathrm{O}(11) & 3,091(7) & \mathrm{O}(8)-\mathrm{O}(72) & 2,630(11) \\ \mathrm{Se}(2)-\mathrm{O}(8) & 1,657(9) & \mathrm{O}(51)-\mathrm{O}(72) & 2,621(11) \\ \mathrm{Se}(2)-\mathrm{O}(72) & 1,695(8) & \mathrm{O}(4)-\mathrm{O}(41) & 2,767(11) \\ \mathrm{Se}(2)-\mathrm{O}(51) & 1,822(8) & \mathrm{O}(4)-\mathrm{O}(1) & 2,741(10) \\ \mathrm{Se}(2)-\mathrm{O}(73) & 2,952(8) & \mathrm{O}(7)-\mathrm{O}(71) & 2,770(11) \\ \mathrm{Se}(2)-\mathrm{O}(2) & 3,018(6) & \mathrm{O}(7)-\mathrm{O}(2) & 2,747(10) \\ \mathrm{V}(1)-\mathrm{O}(1) & 1,619(7) & \mathrm{O}(6)-\mathrm{O}(82) & 2,709(12) \\ \mathrm{V}(1)-\mathrm{O}(4) & 1,958(8) & \mathrm{O}(6)-\mathrm{O}(81) & 2,788(12) \\ \mathrm{V}(1)-\mathrm{O}(11) & 2,313(7) & \mathrm{O}(6)-\mathrm{O}(3) & 2,750(9) \\ \mathrm{V}(2)-\mathrm{O}(2) & 1,605(6) & \mathrm{O}(81)-\mathrm{O}(3) & 2,700(9) \\ \mathrm{V}(2)-\mathrm{O}(7) & 1,985(8) & \mathrm{O}(6)-\mathrm{O}(31) & 2,809(9) \\ \mathrm{V}(2)-\mathrm{O}(21) & 2,327(6) & \mathrm{O}(81)-\mathrm{O}(31) & 2,800(9) \\ \mathrm{O}(4)-\mathrm{Se}(1)-\mathrm{O}(51) & 96,0(4) & \mathrm{O}(41)-\mathrm{V}(1)-\mathrm{O}(4) & 89,9(3) \\ \mathrm{O}(4)-\mathrm{Se}(1)-\mathrm{O}(6) & 100,5(4) & \mathrm{O}(2)-\mathrm{V}(2)-\mathrm{O}(7) & 99,3(3) \\ \mathrm{O}(51)-\mathrm{Se}(1)-\mathrm{O}(6) & 100,6(4) & \mathrm{O}(71)-\mathrm{V}(2)-\mathrm{O}(7) & 8,3(5) \\ \mathrm{O}(51)-\mathrm{Se}(2)-\mathrm{O}(72) & 96,3(4) & \mathrm{O}(3)-\mathrm{V}(3)-\mathrm{O}(81) & 77,8(3) \\ \mathrm{O}(51)-\mathrm{Se}(2)-\mathrm{O}(8) & 100,6(4) & \mathrm{O}(3)-\mathrm{V}(3)-\mathrm{O}(6) & 78,9(3) \\ \mathrm{O}(72)-\mathrm{Se}(2)-\mathrm{O}(8) & 103,4(4) & \mathrm{O}(82)-\mathrm{V}(3)-\mathrm{O}(6) & 86,1(3) \\ \mathrm{Se}(1)-\mathrm{O}(51)-\mathrm{Se}(2) & 119,6(3) & \mathrm{O}(81)-\mathrm{V}(3)-\mathrm{O}(6) & 89,2(3) \\ \mathrm{O}(1)-\mathrm{V}(1)-\mathrm{O}(4) & 99,6(3) & \mathrm{O}(81)-\mathrm{O}(6)-\mathrm{O}(82) & 88,8(3)\end{array}$

* La liste des facteurs de structure a été déposée à la British Library Lending Division (Supplementary Publication No. SUP 30577: $3 \mathrm{pp}$.). On peut en obtenir des copies en s'addressant à: The Executive Secretary, International Union of Crystallography, 13 White Friars, Chester CH1 1 NZ, Angleterre. 


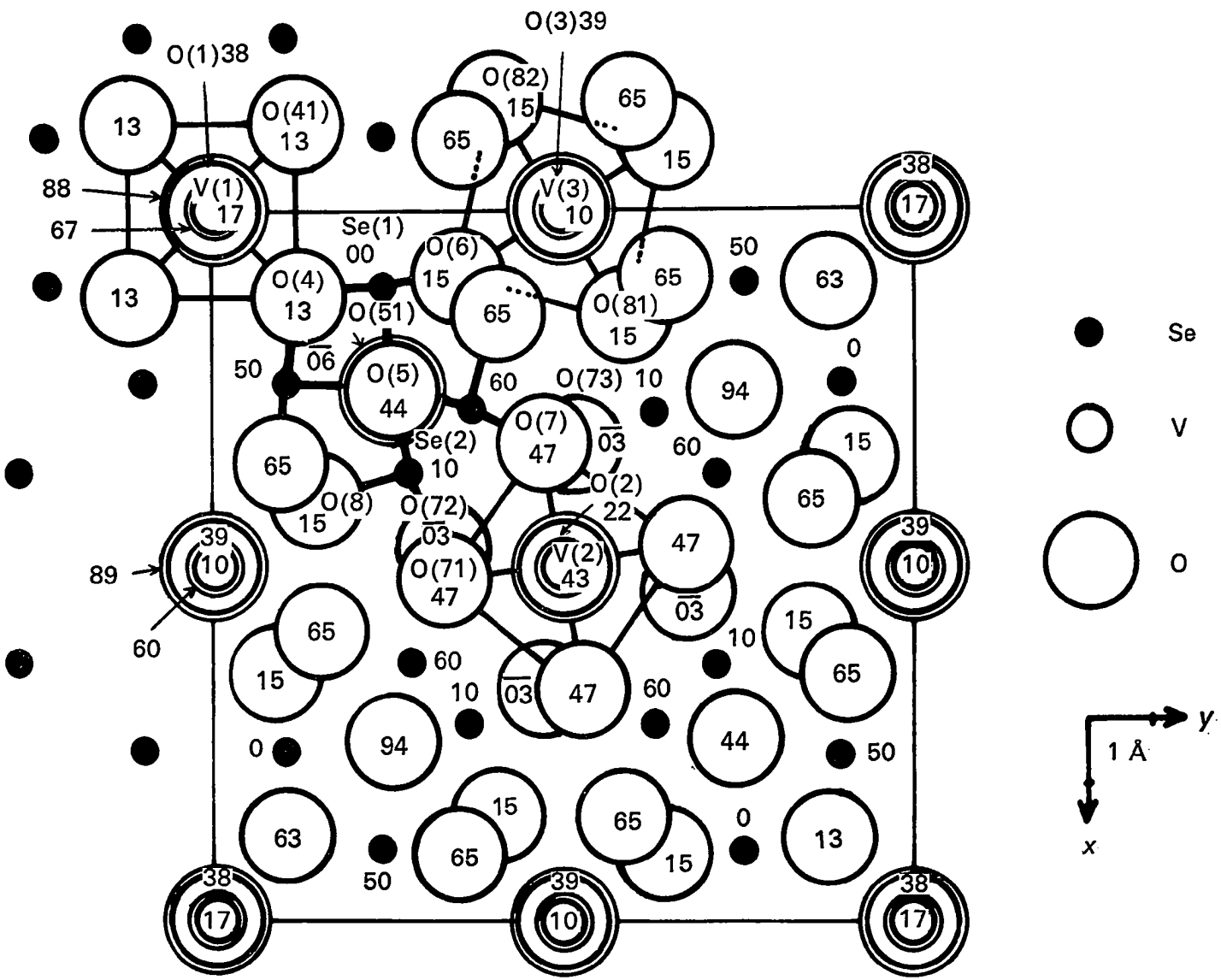

Fig. 1. Projection de la structure de $\mathrm{VSe}_{2} \mathrm{O}_{6}$ sur le plan (001).

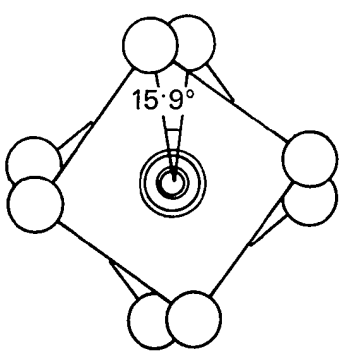

$\left[\mathrm{V}(2) \mathrm{O}_{5}\right]$

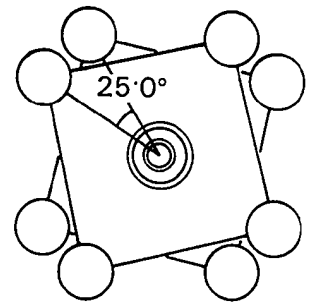

$\left[\mathrm{V}(3) \mathrm{O}_{5}\right]$
Fig. 2. Distorsion des chaînes $V(2)$ et $V(3)$.

Les positions atomiques et les facteurs d'agitation thermique isotrope des différents atomes sont rassemblés au Tableau 4.

Les distances interatomiques et les principaux angles de liaison figurent au Tableau 5.

\section{Description de la structure}

La projection de la structure est donnée à la Fig. 1.

Le réseau cristallin de $\mathrm{VSe}_{2} \mathrm{O}_{6}$ peut se décrire comme résultant de l'assemblage de:
- trois chaînes $\left(\mathrm{VO}_{5}\right)_{n}^{6 n}$ - indépendantes, centrées sur les atomes de vanadium $\mathrm{V}(1), \mathrm{V}(2)$ et $\mathrm{V}(3)$;

- groupements $\left(\mathrm{Se}_{2} \mathrm{O}\right)^{6+}$ qui pontent les chaînes entre elles.

\section{Chaînes $\left(\mathrm{VO}_{5}\right)_{n}^{6 n-}$}

Ces chaînes infinies, parallèles à la direction [001], sont formées d'octaèdres $\mathrm{VO}_{6}$ quasi parfaits liés entre eux par mise en commun de sommets. La périodicité le long de ces chaînes correspond à deux fois la hauteur d'un octaèdre $(2 \times 3,932 \AA)$. On peut noter que la chaîne $\left[\mathrm{V}(1) \mathrm{O}_{5}\right]$ est parfaitement régulière, tous les octaèdres se succédant selon la direction [001] se superposent parfaitement; les oxygènes du plan médian de deux octaèdres consécutifs forment un prisme à base carrée d'axe $O z$. Par contre les chaînes [V(2) $\left.\mathrm{O}_{5}\right]$ et $\left[\mathrm{V}(3) \mathrm{O}_{5}\right]$ présentent une certaine distorsion par suite de légères rotations alternées des octaèdres autour de l'axe de la chaîne; cette distorsion est nettement plus accusée pour la chaîne $\left[\mathrm{V}(3) \mathrm{O}_{5}\right]$, soit $25,0^{\circ}$, que pour la chaîne $\left[\mathrm{V}(2) \mathrm{O}_{5}\right]$, soit $15,9^{\circ}$ (Fig. 2).

Les atomes de vanadium V(1), V(2) et V(3) n'occupent pas le centre des octaèdres mais s'écartent du plan médian, dans la direction [001] pour $V(1)$ et $[00 \overline{1}]$ pour $V(2)$ et $V(3)$, pour former avec l'oxygène du 


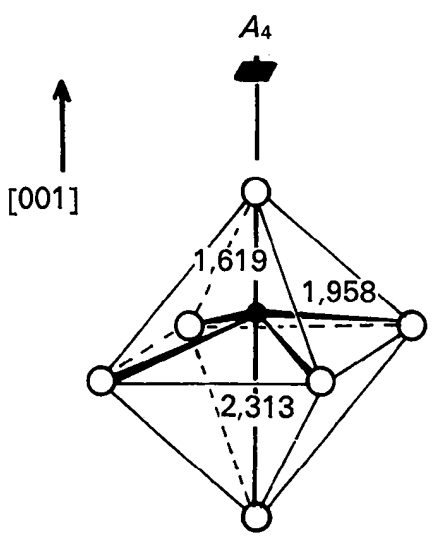

$\mathrm{V}(1) \mathrm{O}_{6}$

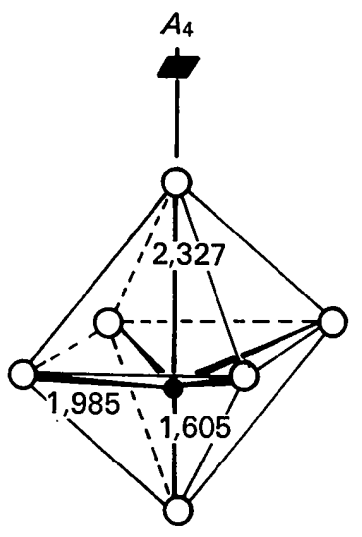

$\mathrm{V}(2) \mathrm{O}_{6}$

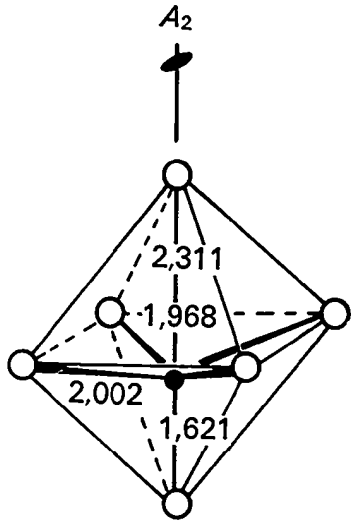

$\mathrm{V}(3) \mathrm{O}_{6}$

Fig. 3. Déplacement des atomes de vanadium dans les files $\left[\mathrm{V}(1) \mathrm{O}_{5}\right]_{n}^{6 n-},\left[\mathrm{V}(2) \mathrm{O}_{5}\right]_{n}^{6 n-}$ et $\left[\mathrm{V}(3) \mathrm{O}_{5}\right]_{n}^{6 n-}$.

sommet de l'octaèdre une liaison $\mathrm{V}-\mathrm{O}$ de valeur moyenne $\mathrm{V}-\mathrm{O}=1,615 \AA$, typique du radical vanadyle $\mathrm{VO}^{2+}$ (Fig. 3). Les chaînes $\left[\mathrm{V}(2) \mathrm{O}_{5}\right]$ et $\left[\mathrm{V}(3) \mathrm{O}_{5}\right]$ apparaissent donc comme 'antiparallèles' avec la chaîne $\left[\mathrm{V}(1) \mathrm{O}_{5}\right]$.

\section{Groupements $\left[\mathrm{Se}_{2} \mathrm{O}\right]^{6+}$}

Chaque atome de sélénium, $\mathrm{Se}(1)$ et $\mathrm{Se}(2)$, est lié à trois atomes d'oxygène formant ainsi une pyramide à base triangulaire $\mathrm{SeO}_{3}$. Deux des atomes d'oxygène de cette pyramide appartiennent aux chaînes $\left(\mathrm{VO}_{5}\right)$ $\left\{\left[\mathrm{V}(1) \mathrm{O}_{5}\right]\right.$ et $\left.\left[\mathrm{V}(3) \mathrm{O}_{5}\right)\right]$ pour $\mathrm{Se}(1)$ et $\left.\left[\mathrm{V}(3) \mathrm{O}_{5}\right)\right]$ et $\left.\left[\mathrm{V}(2) \mathrm{O}_{5}\right)\right]$ pour $\left.\mathrm{Se}(2)\right\}$, le troisième, $\mathrm{O}(5)$, partage deux liaisons avec les seuls atomes $\mathrm{Se}(1)$ et $\mathrm{Se}(2) ; \mathrm{Se}(1)$ $\mathrm{O}-\mathrm{Se}(2)$ forment un groupement $\left[\mathrm{Se}_{2} \mathrm{O}\right]^{6+}$

Si l'on considère le quart de maille, les quatre chaînes diagonalement opposées, $\left.\left[\mathrm{V}(1) \mathrm{O}_{5}\right)\right]$ et $\left[\mathrm{V}(2) \mathrm{O}_{5}\right]$ et les deux $\left[\mathrm{V}(3) \mathrm{O}_{5}\right]$, sont liées entre elles par deux groupements $\left[\mathrm{Se}_{2} \mathrm{O}\right]^{6+}$ ou plus précisément $[\mathrm{Se}(1)-$ $\mathrm{O}(5)-\mathrm{Se}(2)]$ et $\left[\mathrm{Se}\left(1^{\prime}\right)-\mathrm{O}\left(5^{\prime}\right)-\mathrm{Se}\left(2^{\prime}\right)\right]$ d'orientations moyennes [100] et [010].

\section{Coordinence du sélénium(+IV)}

Stéréochimie du groupe $\left(\mathrm{Se}_{2} \mathrm{O}_{5}\right)^{2-}$

Comme nous l'avons signalé précédemment les données de la littérature sur la cristallochimie des composés oxygénés du sélénium( $+I V)$ sont peu nombreuses. Les longueurs de liaison trouvées dans $\mathrm{VSe}_{2} \mathrm{O}_{6}$ sont en accord avec celles publiées par McCullough (1937) pour $\mathrm{SeO}_{2}\left(\mathrm{Se}-\mathrm{O}_{\text {moy. }}=1,76 \AA\right)$ et par Gattow (1958) pour $\mathrm{CuSeO}_{3} \cdot 2 \mathrm{H}_{2} \mathrm{O}\left(\mathrm{Se}-\mathrm{O}_{\text {moy. }}=\right.$ $1,76 \AA$ ).

Le rôle joué par le doublet $4 s_{5}$ du sélénium(+IV) est considérable; les effets stéréochimiques de tels doublets $\left(4 s^{2}, 5 s^{2}\right.$ et $\left.6 s^{2}\right)$ ont été précisés par Andersson et al. (1973) et par Galy, Meunier, Andersson \& Åström (1974).

Le sélénium peut être considéré comme étant à

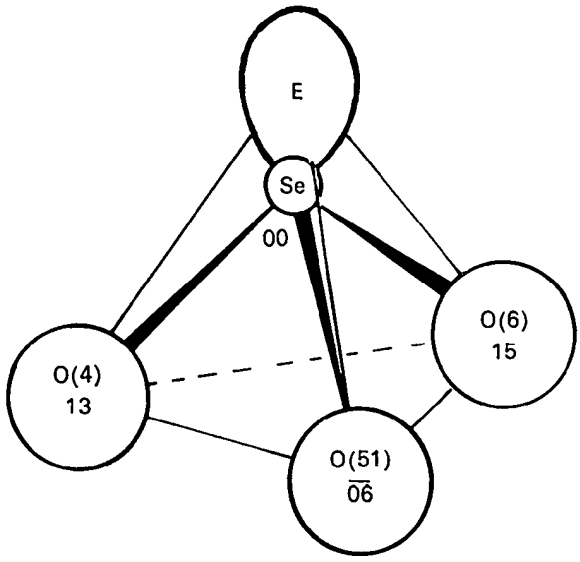

Fig. 4. Coordinence du sélénium(+IV). ( $E$ symbolise la paire non liée).

l'intérieur d'une pyramide à base triangulaire formée par trois oxygènes la paire non liée $E$ étant située à l'apex (Fig. 4).

La structure de la phase $\mathrm{VSe}_{2} \mathrm{O}_{6}$ présente quelques caractères originaux quant à l'association de tels groupes $\mathrm{SeO}_{3} E$. On peut en effet décrire de manière différente la structure de $\mathrm{VSe}_{2} \mathrm{O}_{6}$ en faisant ressortir la présence de groupements $\left(\mathrm{Se}_{2} \mathrm{O}_{5}\right)^{2-}$ au sein du réseau. Ces

$$
\text { groupements }\left(\mathrm{Se}_{2} \mathrm{O}_{5}\right)^{2-}, \mathrm{Se}(1)-\mathrm{O}(5)-\mathrm{Se}(2)
$$

s'agencent de telle manière qu'ils ménagent au sein du réseau trois tunnels oxygénés parallèles à l'axe $O z[\mathrm{~V}(1)$, $\mathrm{V}(2)$ et $\mathrm{V}(3)$ ] dans lesquels viennent s'insérer les groupements vanadyles $\mathrm{VO}^{2+}$.

Ces groupes discrets $\left(\mathrm{Se}_{2} \mathrm{O}_{5}\right)^{2-}$ peuvent être comparés aux groupes $\left(\mathrm{Sn}_{2} \mathrm{~F}_{5}\right)^{-}$décrits par McDonald, 

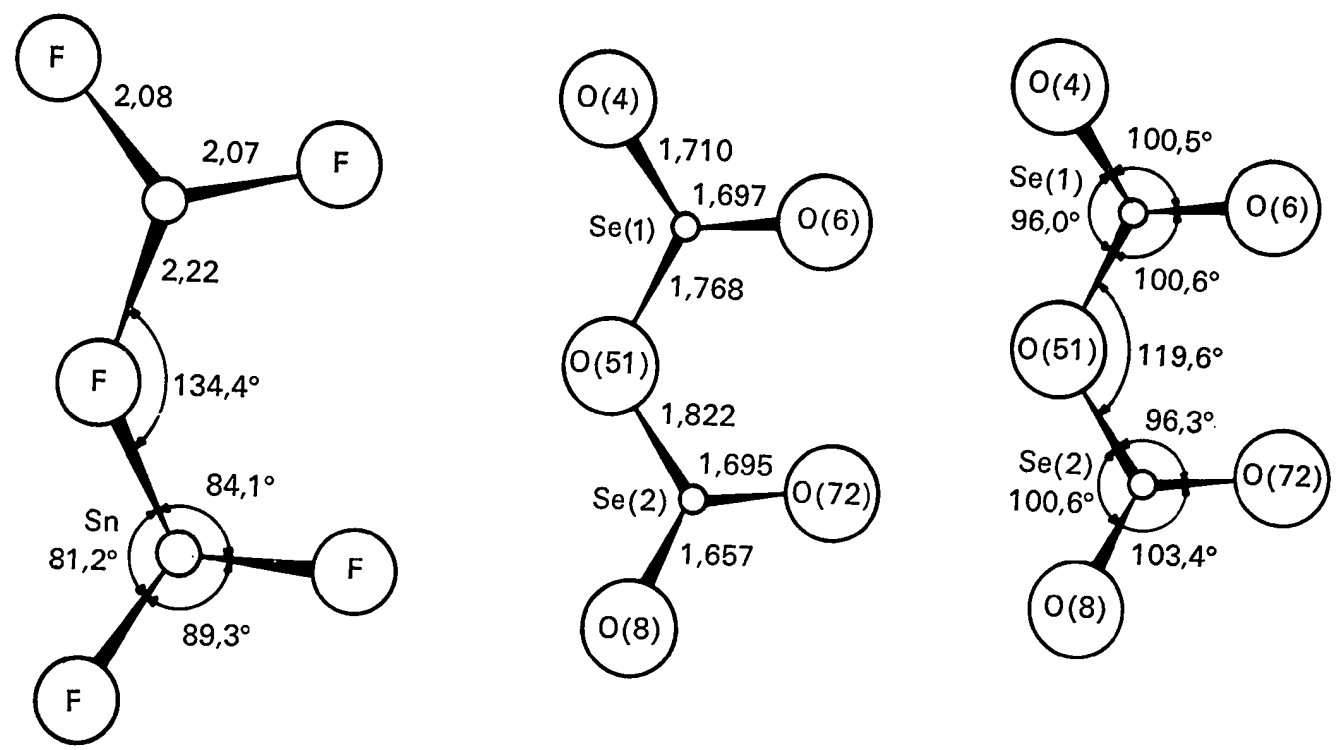

Fig. 5. Comparaison entre les groupements $\left(\mathrm{Sn}_{2} \mathrm{~F}_{5}\right)^{-}$et $\left(\mathrm{Se}_{2} \mathrm{O}_{5}\right)^{2-}$.

Larson \& Cromer (1964), qui caractérisent le réseau du fluorure double d'étain et de sodium $\mathrm{NaSn}_{2} \mathrm{~F}_{5}$ (Fig. 5). On peut noter dans les deux groupes $\left(\mathrm{Sn}_{2} \mathrm{~F}_{5}\right)^{-}$ et $\left(\mathrm{Se}_{2} \mathrm{O}_{5}\right)^{2-}$ que les liaisons $\mathrm{Sn}-\mathrm{F}(2,07$ et $2,08 \AA)$ et Se-O $(1,710,1,697,1,657$ et 1,695 $\AA$ ) terminales sont plus courtes que la liaison correspondant au pont $\mathrm{Sn}-\mathrm{F}-\mathrm{Sn} \quad(2,22 \AA)$ ou $\mathrm{Se}(1)-\mathrm{O}(5)-\mathrm{Se}(2) \quad(1,768$ et $1,822 \AA$ ).

De nouvelles investigations actuellement en cours sur des composés inédits du sélénium(+IV) permettront de préciser la stéréochimie particulièrement originale de cet élément.

\section{Références}

Andersson, S., Åström, A., Galy, J. \& Meunier, G. (1973). J. Solid State Chem. 6, 187-190.

Charlot, G. (1961). Les Méthodes de la Chimie Analytique, 4ème éd., pp. 889, 962.
GaLY, J. (1972). N.B.S. Special Publication 364, Solid State Chem. pp. 29-39.

Galy, J. \& Meunier, G. (1971). Acta Cryst. B27, 608-616. Galy, J., Meunier, G., Andersson, S. \& Åström, A. (1974). J. Solid State Chem. A paraître.

Gattow, G. (1958). Acta Cryst. 11, 377-383.

Howells, E. R., Philips, C. D. \& Rogers, D. (1950). Acta Cryst. 3, 210-214.

McCullough, J. D. (1937). J. Amer. Chem. Soc. 59, 789 794.

McDonald, R. R., Larson, A. C. \& Cromer, D. T. (1964). Acta Cryst. 17, 1104-1108.

McMaster, W. H., KerR del Grande, N., Mallet, J. H. \& HubBel, H. J. (1969). N.B.S. Compilation of X-ray Cross Sections, UCRL-50174, Sec. II, Rev. 1.

Meunier, G., Darriet, J. \& Galy, J. (1972). J. Solid State Chem. 5, 314-320.

Meunier, G., Darriet, J. \& Galy, J. (1973). J. Solid State Chem. 6, 67-73.

Meunier, G. \& Galy, J. (1971). Acta Cryst. B27, 602-608.

Srinivasan, R. (1960). Acta Cryst. 13, 388-394.

Wilson, A. J. C. (1949). Acta Cryst. 2, 318-321. 